\title{
Wiretap Codes for Secure Multi-Party Computation
}

\author{
Ragnar Thobaben, György Dán, and Henrik Sandberg \\ KTH Royal Institute of Technology and ACCESS Linnaeus Centre, \\ SE-100 44 Stockholm, Sweden \\ E-mail: \{ragnart, gyuri, hsan\}@kth.se
}

\begin{abstract}
In this paper, we propose a new secret sharing scheme for secure multi-party computation. We present a general framework that allows us to construct efficient secret sharing schemes from channel coding techniques for the wiretap channel. The resulting schemes can be employed to securely calculate linear functions of data that are distributed in a network without leaking any information on the data except the desired result. For the examples considered in this paper, our schemes minimize the communication overhead while keeping the data perfectly secure. Compared to conventional schemes, for which the communication overhead grows quadratically in the number of clients in the considered scenarios, the communication overhead for our approach grows only linearly with the number of clients. This property is maintained even if our secret sharing scheme is set up to introduce redundancy in order to compensate for losses of secret shares. While we only consider the case of passive eavesdroppers and implementations based on nested Reed-Solomon codes in this paper, the proposed framework can also be applied in other cases (e.g., when clients tamper with the data) by taking into account the effects of attacks in the design of the underlying wiretap code.
\end{abstract}

\section{INTRODUCTION}

Secure multi-party computation has recently gained significant attention since it has the potential to resolve privacy issues in many application areas. For example, secure data aggregation protocols can be employed for smart metering in order to collect real-time readings of the overall energy consumption of a group of customers without leaking the instantaneous energy consumption of individual customers [1]. Protocols for secure multi-party computation are typically based on secret sharing. That is, in order to keep their data private clients decompose their data into random shares which are distributed among the clients in the network in order to carry out the desired computations. In some cases, e.g., if a collecting node is also involved in the computation, protocols also employ additively homomorphic public-key encryption like, e.g., the Paillier crypto system [2]. Applications of homomorphic encryption to secure signal processing have recently been discussed in [3]. Unfortunately, many of the proposed solutions lead to a significant communication overhead, which in many examples grows quadratically with the number of clients that are involved in the computation.

In this paper, we propose a new secret sharing scheme that significantly reduces the size of the secret shares such that the overall communication overhead scales linearly with the number of clients. Our scheme is constructed from nested codes for the wiretap channel [4]. Due to the linearity of the underlying code designs, this approach is well suited for computation of linear functions of data that are distributed in a network, and it shows the same capabilities as currently considered additively homomorphic crypto systems. Our scheme is parameterized by the total number of secret shares that are generated and two design thresholds: the number of secret shares that are required to reconstruct the secret and the maximum number of secret shares that can be published without leaking any information. The result of this approach are secret sharing schemes that are highly efficient in the sense that they minimize the communication overhead while keeping the data perfectly secure. The good performance of our schemes is demonstrated in three different examples and compared to standard secret sharing protocols.

In this paper, we only consider the case of passive eavesdroppers and discuss implementations based on (punctured) Reed-Solomon codes (see, e.g., [5]) that allow for highly efficient and perfectly secure secret sharing with finite block length. Since our secret sharing scheme is constructed from linear codes it may also be extended to other attacker models where clients tamper with the secret shares of other clients. This can be achieved by adopting, e.g., polar code designs (see, e.g., [6]-[8]) to the resulting wiretap channel model. However, for finite block length, this approach would not be able to guarantee perfect secrecy.

We note that the relation between secret sharing and ReedSolomon coding has been recognized before in [9] where McEliece and Sarwate point out that Shamir's $(k, n)$ threshold scheme [10] can be interpreted as a special case of ReedSolomon coding. In [9], the authors also propose to utilize code properties to perform data compression. However, their approach suffers from unavoidable information leakage whereas our scheme is explicitly designed to provide data compression capabilities while maintaining perfect secrecy. Reed-Solomon wiretap codes have furthermore been used for secure network coding, e.g., in [11]. In recent research on secret sharing (e.g., [12]-[14]), efficient schemes for secret sharing in the presence of cheaters have been presented, which however also employ cryptographic techniques like hashing and message authentication codes.

The remainder of this paper is organized as follows: In Section II we present the general problem formulation, summarize basic secret sharing and secure multi-party computation protocols, and introduce the nested coding scheme for the wiretap channel that is the basis for our secret sharing scheme. The proposed scheme is discussed in Section III, and the good performance of the scheme is verified in the examples in Section IV. We summarize our conclusions in Section V.

\section{PRELIMINARIES}

\section{A. Problem Formulation}

The setup considered in this paper consists of a set of $K$ clients $\left\{\mathrm{C}_{0}, \ldots, \mathrm{C}_{K-1}\right\}$, which hold individual data 
$\boldsymbol{m}_{0}, \ldots, \boldsymbol{m}_{K-1}$. The clients are connected through a network with public and/or private links. The goal is to calculate linear functions of the data in a distributed fashion and to share the result while keeping the individual data private. We assume that the data $\boldsymbol{m}_{i}$ are vectors of length $L_{m}$, defined over the finite field $\mathcal{F}_{q}$, where $q$ is a prime or a power of a prime. The elements in $\boldsymbol{m}_{i}$ are assumed to be i.i.d., drawn from a uniform distribution over the field $\mathcal{F}_{q}$. In the remainder of this paper, we assume that all arithmetic operations on the data are carried out on the field $\mathcal{F}_{q}$.

\section{B. Secret Sharing}

Due to the constraint that the individual data must be kept private, secure multi-party computation relies heavily on secret sharing. In the following, we introduce a baseline scheme and Shamir's $(k, n)$ threshold scheme. For the latter, we explain the connection to Reed-Solomon (RS) coding. For ease of notation, we restrict our discussion to the scalar case; however, all schemes can easily be extended to the vector case by applying them elementwise. A protocol that shows how secret sharing can be applied for multi-party computation is presented in Section II-C.

1) Baseline Scheme: The goal of this scheme is to share information about secret data $m$ without revealing it. To achieve this a client decomposes the data $m$ into a sum of $k$ random shares $m^{(j)}$,

$$
m=\sum_{j=1}^{k} m^{(j)},
$$

where we assume that the shares $m^{(j)}$ for $j \in\{1, \ldots, k-1\}$ are independently drawn from a uniform distribution over the field $\mathcal{F}_{q}$ and $m^{(k)}$ is chosen to satisfy Eq. (1). The shares can then be distributed among the cooperating clients to initiate the computation of the desired function of the data. Note that $m^{(k)}$ follows as well a uniform distribution over $\mathcal{F}_{q}$. From this, it follows that $m$ is perfectly secure as long as at most $k-1$ shares become public.

The baseline scheme suffers from two drawbacks: (i) it increases the communication overhead by a factor of $k$, and (ii) if a share is lost, the secret cannot be recovered. This becomes an issue if clients are not permanently online in the network (e.g., due to sleep cycles).

2) Shamir's $(k, n)$ Threshold Scheme [10]: To make secret sharing robust against losses of shares, Shamir introduced a $(k, n)$ threshold scheme with $k \leq n<q$ that splits the secret $m$ into $n$ shares such that (i) the secret can be reconstructed by any subset of $k$ shares and (ii) the secret is perfectly secure as long as $k-1$ (or fewer) shares are public. We note that the baseline scheme above is a $(k, k)$ threshold scheme.

Shamir's scheme employs a degree- $(k-1)$ random polynomial $p(x)=a_{0}+a_{1} x+\ldots+a_{k-1} x^{k-1}$ with $a_{0}=m$. The remaining coefficients $a_{i}$ are drawn from a uniform distribution over the $\mathcal{F}_{q}$, and the secret can be recovered from the polynomial by evaluating $p(0)$. The shares $m^{(j)}$ for $j \in\{1, \ldots, n\}$ are then obtained as $m^{(j)}=p(j)$.

The first property is satisfied due to the fact that $p(x)$ is a polynomial of degree $(k-1)$ which can be recon- structed by interpolation from any set of $k$ values of the polynomial $\left\{p\left(x_{1}\right), \ldots, p\left(x_{k}\right)\right\}$ evaluated in $k$ distinct positions $x_{1}, \ldots, x_{k}$. If now $(k-1)$ shares are public, an attacker can reconstruct $q$ equally likely polynomials $p^{\prime}(x)$ of which only one satisfies $p^{\prime}(0)=m$ such that no information on $m$ leaks out. Unfortunately, due to the additional redundancy, Shamir's scheme leads to an additional increase in the communication overhead since now up to $n$ shares are distributed.

As pointed out in [9], Shamir's scheme can be interpreted as a special case of RS coding. Here, the coefficients $a_{i}$ of the polynomial $p(x)$ correspond to the information symbols, the shares $m^{(j)}$ correspond to code symbols of a rate- $k / n$ RS code (a standard RS code if $n=q-1$, and a punctured RS code if $n<q-1$ ), and the $j$-th column $\mathbf{g}_{j}$ of the generator matrix $\mathbf{G}$ of the $\mathrm{RS}$ code is given by $\mathbf{g}_{j}=\left[1, j^{2}, \ldots, j^{k-1}\right]^{T}$.

\section{A Secure Data Aggregation Protocol}

We consider a scenario where $K$ clients form a fully connected network in order to calculate the sum of their data

$$
\boldsymbol{m}=\sum_{i=0}^{K-1} \boldsymbol{m}_{i}
$$

without revealing the individual data $\boldsymbol{m}_{i}$. The links between the clients are assumed to be secure and authenticated. To simplify the discussion, we consider only the case where the baseline secret sharing scheme is employed. The protocol can however be extended by applying Shamir's scheme elementwise and by utilizing the linearity of the underlying RS code.

In a first step, each client $\mathrm{C}_{i}$ generates $k=K$ random shares $\boldsymbol{m}_{i}^{(j)}$ of its data $\boldsymbol{m}_{i}$. For every $j \neq i$, each client $\mathrm{C}_{i}$ sends then the share $\boldsymbol{m}_{i}^{(j)}$ to $\mathrm{C}_{j}$ by using the secure link between $\mathrm{C}_{i}$ and $\mathrm{C}_{j}$ and keeps $\boldsymbol{m}_{i}^{(i)}$ private. After all shares are distributed, each client $\mathrm{C}_{i}$ holds a set of shares $\left\{\boldsymbol{m}_{0}^{(i)}, \ldots, \boldsymbol{m}_{K-1}^{(i)}\right\}$ and calculates the intermediate sum

$$
\boldsymbol{m}^{(i)}=\sum_{j=0}^{K-1} \boldsymbol{m}_{j}^{(i)} \text {. }
$$

The intermediate sums $\boldsymbol{m}^{(i)}$ are finally broadcasted by the clients by using a public broadcast channel. For the baseline scheme (after receiving the broadcasted shares), every client calculates locally the desired result

$$
\boldsymbol{m}=\sum_{i=0}^{K-1} \boldsymbol{m}^{(i)}
$$

Even though, only $K-1$ shares are shared by the clients in this protocol, it is essential to require secure links between the clients since otherwise, the individual messages can be recovered from the intermediate sums $\boldsymbol{m}^{(i)}$. In total, $K^{2}$ transmissions and a communication overhead of $N_{B L}=K^{2} L_{m}$ symbols are required to calculate and distribute the result.

\section{Channel Coding for the Wiretap Channel}

The secret sharing scheme proposed in this paper will make use of nested linear codes for the wiretap channel [4]. The wiretap channel model consists of a pair of transmitter and 
receiver, Alice and Bob, respectively, that communicate in the presence of an eavesdropper Eve. The goal is to establish reliable communication between Alice and Bob while keeping Eve uninformed. Here, we are interested in a special class of wiretap erasure channels for which the numbers of erasures seen by Bob and Eve are constant for a given block length (as opposed to channels that are characterized by an average erasure probability). As shown in [5], wiretap codes that achieve the secrecy capacity under perfect secrecy constraint for this class of channels can be constructed from RS codes. That is, denoting the message to be transmitted as $\boldsymbol{m}$ and the observations at Bob and Eve as $\boldsymbol{y}_{B}$ and $\boldsymbol{y}_{E}$, respectively, we can guarantee that

$$
\begin{array}{lr}
H\left(\boldsymbol{M} \mid \boldsymbol{Y}_{B}\right)=0 & \text { (reliability) } \\
H\left(\boldsymbol{M} \mid \boldsymbol{Y}_{E}\right)=H(\boldsymbol{M}) & \text { (perfect secrecy). }
\end{array}
$$

The code construction is summarized in the following.

In general, wiretap codes can be constructed from a pair of nested linear codes $\left\{\mathcal{C}_{0}, \mathcal{C}\right\}$ with $\mathcal{C} \subset \mathcal{C}_{0}$ and rates $\left(R_{0}, R^{\prime}\right)$ with $R_{0}>R^{\prime}$. In the following, let $\boldsymbol{H}_{0}$ be the parity check matrix of the fine code $\mathcal{C}_{0}$ with dimensions $\left(k_{0} \times n\right)$ and let $\boldsymbol{H}=\left[\boldsymbol{H}_{0}^{T} \boldsymbol{H}_{1}^{T}\right]^{T}$ be the parity check matrix of the coarse code $\mathcal{C}$. The check matrix $\boldsymbol{H}_{1}$ has dimension $(k \times n)$. The fine code $\mathcal{C}_{0}$ is now split into disjoint subcodes $\mathcal{C}(i)$ that are realized as cosets of the coarse code $\mathcal{C}$. The number of subcodes is chosen to equal the size of the message set. For encoding, the message $\boldsymbol{m}$ determines the subcode $\mathcal{C}(\boldsymbol{m})$ that is used for the transmission, and Alice randomly selects a codeword $\boldsymbol{x}$ from the subcode $\mathcal{C}(\boldsymbol{m})$ that is sent over the channel. The underlying randomized coset coding scheme for encoding the length- $k$ message $\boldsymbol{m}$ can then be described as picking a random solution $\boldsymbol{x}$ that satisfies

$$
\underbrace{\left[\begin{array}{c}
\boldsymbol{H}_{0} \\
\boldsymbol{H}_{1}
\end{array}\right]}_{\doteq \boldsymbol{H}} \boldsymbol{x}^{T}=\left[\begin{array}{c}
\mathbf{0} \\
\boldsymbol{m}^{T}
\end{array}\right] .
$$

As long as the fine code is decodable over the main channel, Bob can decode the transmitted codeword $\boldsymbol{x}$ (e.g., by using the check matrix $\boldsymbol{H}_{0}$ ) and recover the message by utilizing the fact that $\boldsymbol{H}_{1} \boldsymbol{x}^{T}=\boldsymbol{m}^{T}$. The rate of (secure) communication is given by $R=R_{0}-R^{\prime}=k / n$.

For transmission over the wiretap erasure channel with constant erasure probabilities, the optimal choice of code parameters is summarized in the following lemma (see as well [5]):

Lemma 1: Consider a transmission over a wiretap erasure channel with $n$ channel uses and $e_{B}$ and $e_{E}$ erasures on the channels to Bob and Eve, respectively, using the coset-coding scheme from Eq. (2), where $\mathcal{C}_{0}$ and $\mathcal{C}$ are a pair of (punctured) nested Reed-Solomon codes defined over the field $\mathcal{F}_{q}$. For $k_{0}=e_{B}$ and $k=e_{E}-e_{B}$, reliability and perfect secrecy are guaranteed and the secrecy rate $R$ equals the secrecy capacity $C_{s}=\left(e_{E}-e_{B}\right) / n$.

Proof: Consider first the case $n=q-1$. For $k_{0}=e_{B}$, reliability follows immediately from the MDS (maximumdistance separable) property of the fine code $\mathcal{C}_{0}$. Furthermore, from Lemma IV.1 in [6] we know that

$$
\begin{aligned}
H\left(\boldsymbol{M} \mid \boldsymbol{Y}_{E}\right) & =H\left(\boldsymbol{X} \mid \boldsymbol{Y}_{E}\right)-H\left(\boldsymbol{X} \mid \boldsymbol{Y}_{E}, \boldsymbol{M}\right) \\
& =\left(\operatorname{rank}\left\{\boldsymbol{H}_{\mathcal{E}}\right\}-\operatorname{rank}\left\{\boldsymbol{H}_{0 \mathcal{E}}\right\}\right) \log (q),
\end{aligned}
$$

where $\boldsymbol{H}_{\mathcal{E}}$ and $\boldsymbol{H}_{0 \mathcal{E}}$ are the matrices formed from the columns of $\boldsymbol{H}$ and $\boldsymbol{H}_{0}$, respectively, that correspond to erased codeword positions at the eavesdropper. For $k_{0}=e_{B}$ and $k=e_{E}-e_{B}$, perfect secrecy follows from the MDS property of the underlying codes, i.e.,

$$
H\left(\boldsymbol{M} \mid \boldsymbol{Y}_{E}\right)=\left(\left(k_{0}+k\right)-k_{0}\right) \log (q)=k \log (q)=H(\boldsymbol{M}),
$$

and the secrecy rate $R$ equals the secrecy capacity $C_{s}=\left(e_{E}-\right.$ $\left.e_{B}\right) / n$. The proof for $n<q-1$ follows immediately from the fact that punctured MDS codes are MDS codes as well.

Note that the structure shown in Eq. (2) is not the only structure for generating nested codes. Alternatively, one can define nested codes by splitting the message set into disjoint subsets. In this way, Shamir's $(k, n)$ threshold scheme can also be interpreted as a secret sharing scheme constructed from a wiretap code, whereas the extension by McEliece and Sarwate in [9] does neither define a nested code structure nor uses a randomized encoder.

\section{SEcret Sharing With Wiretap Codes}

\section{A. Secret Sharing with Wiretap Codes}

We propose a secret sharing scheme that employs the wiretap codes introduced in Section II-D to generate the secret shares. In our scheme, the data $\boldsymbol{m}_{i}$ of the clients play the role of messages that are encoded with a wiretap code. In order to generate a set of secret shares, client $\mathrm{C}_{i}$ encodes its data $\boldsymbol{m}_{i}$ by a codeword $\boldsymbol{x}_{i}$ of a wiretap code $\left\{\mathcal{C}_{0}, \mathcal{C}\right\}$, with rate assignment $\left(R_{0}, R^{\prime}\right)$ and secrecy rate $R=R_{0}-R^{\prime}$ and splits the codeword $\boldsymbol{x}_{i}$ into $L$ packets $\boldsymbol{x}_{i}^{(j)}$ of length $\tilde{n}$; i.e., $\boldsymbol{x}_{i}=\left[\boldsymbol{x}_{i}^{(0)}, \ldots, \boldsymbol{x}_{i}^{(L-1)}\right]$ and $n=\tilde{n} L$. To be able to employ the proposed secret sharing scheme for multi-party computation, it is necessary that all clients use the same check matrices $\boldsymbol{H}_{0}$ and $\boldsymbol{H}_{1}$ to generate their secret shares. It is also necessary that the random symbols that are used in the encoding (see Eq. (2)), are independently drawn from a uniform distribution over the field $\mathcal{F}_{q}$, independent among the clients, and kept private at all time.

In order to determine the rate assignment of the employed wiretap code, we introduce two design parameters: the decoding threshold $L_{d}$ and the secrecy threshold $L_{s}$. The decoding threshold $L_{d}$ is defined as the lowest number of packets that are required to reconstruct the secret. In the design of the wiretap code (see Lemma 1), it is related to the number of erasures $e_{B}$ on the main channel as $e_{B}=\tilde{n}\left(L-L_{d}\right)$. The secrecy threshold $L_{s}$ is defined as the maximum number of packets for which the information leakage is guaranteed to be zero. In the design of the wiretap code, it is related to the number of erasures $e_{E}$ in the observation of the eavesdropper as $e_{E}=\tilde{n}\left(L-L_{s}\right)$. The rates of the fine code, the coarse code, and the secrecy rate follow accordingly as $R_{0}=L_{d} / L$, $R^{\prime}=L_{s} / L$, and $R=\left(L_{d}-L_{s}\right) / L$, respectively. The length $L_{m}$ of the data vectors $\boldsymbol{m}_{i}$ and the length $\tilde{n}$ of the secret shares are furthermore related as

$$
L_{m}=n R=\tilde{n}\left(L_{d}-L_{s}\right) .
$$

\section{B. Properties}

The proposed secret sharing scheme features a number of properties that are useful for applications in multi-party computation. It can be designed to be perfectly secure, it preserves 
linearity, its effect on the data vectors can be described similar to the baseline secret sharing scheme from Section II-B1, and depending on the choice of the design parameters, it leads to data compression as we will show at the end of this section.

1) Leakage: Let $L_{E}$ be the number of secret shares that are observed by an adversary. As long as $L_{E} \leq L_{s}$, the underlying wiretap code is used within its specifications and no information leaks out. For $L_{E}>L_{s}$ information leaks out, and the leakage is characterized in the following proposition.

Proposition 2: For $L_{E}>L_{s}$, the average leakage is quantified by the mutual information

$$
I\left(\boldsymbol{M} ; \boldsymbol{Y}_{E}\right)=\tilde{n} \log (q) \cdot \min \left\{L_{d}-L_{s} ; L_{E}-L_{s}\right\} .
$$

Proof: For $L_{s}<L_{E}<L_{d}$, leakage follows directly from Eq. (3). For $L_{E} \geq L_{d}$ the adversary can decode and reconstruct the data.

2) Linearity: The proposed secret sharing scheme preserves linearity in the following sense:

Proposition 3: Let $\boldsymbol{x}_{0}, \boldsymbol{x}_{1}, \ldots$ be codewords encoding the data $\boldsymbol{m}_{0}, \boldsymbol{m}_{1}, \ldots$, respectively. Then, $\boldsymbol{x}_{0}=\sum_{i>0} a_{i} \boldsymbol{x}_{i}$, with coefficients $a_{i} \in \mathcal{F}_{q}$, implies that $\boldsymbol{m}_{0}=\sum_{i>0} a_{i} \boldsymbol{m}_{i}$.

Proof: The statement follows directly from Eq. (2).

3) Equivalence to the Baseline Scheme: In the proposed scheme, secret shares are defined as segments of codewords of a wiretap code. However, it is not immediately clear how this operation maps into operations on the data vectors. The following proposition shows that the segmentation of the codewords implies a decomposition of the data similar to the baseline secret sharing from Section II-B1.

Proposition 4: The decomposition of the wiretap codewords $\boldsymbol{x}_{i}$ into segments $\boldsymbol{x}_{i}^{(0)}, \ldots, \boldsymbol{x}_{i}^{(L-1)}$ implies a decomposition of the data vectors $\boldsymbol{m}_{i}$ into random shares $\boldsymbol{m}_{\boldsymbol{x}_{i}}^{(j)}$, with

$$
\boldsymbol{m}_{i}=\sum_{j=0}^{L-1} \boldsymbol{m}_{\boldsymbol{x}_{i}}^{(j)}
$$

Proof: Let $\boldsymbol{H}_{0}^{(j)}$ and $\boldsymbol{H}_{1}^{(j)}$ denote the sub-matrices in $\boldsymbol{H}_{0}$ and $\boldsymbol{H}_{1}$ that correspond to the $j$-th segment $\boldsymbol{x}_{i}^{(j)}$ in $\boldsymbol{x}_{i}$ which encodes a data vector $\boldsymbol{m}_{i}$. Then,

$$
\left[\begin{array}{c}
\mathbf{0}^{T} \\
\boldsymbol{m}_{i}^{T}
\end{array}\right]=\left[\begin{array}{c}
\boldsymbol{H}_{0}^{(0)} \\
\boldsymbol{H}_{1}^{(0)}
\end{array}\right] \boldsymbol{x}_{i}^{(0)^{T}}+\ldots+\left[\begin{array}{c}
\boldsymbol{H}_{0}^{(L-1)} \\
\boldsymbol{H}_{1}^{(L-1)}
\end{array}\right] \boldsymbol{x}_{i}^{(L-1)^{T}}
$$

Accordingly, each segment $\boldsymbol{x}_{i}^{(j)}$ can be mapped into a secret share $\boldsymbol{m}_{\boldsymbol{x}_{i}}^{(j)}$, with $\boldsymbol{m}_{\boldsymbol{x}_{i}}^{(j)^{T}}=\tilde{\boldsymbol{H}}_{1}^{(j)} \boldsymbol{x}_{i}^{(j)^{T}}$, such that

$$
\sum_{j=0}^{L-1} \boldsymbol{m}_{\boldsymbol{x}_{i}}^{(j)}=\boldsymbol{m}_{i}
$$

4) Data Compression: As we will see in the following, the proposed scheme leads also to a compression of the data compared to the baseline scheme from Section II-B1.

Proposition 5: If the proposed secret sharing scheme is used to replace the baseline scheme from Section II-B1, it will reduce the communication overhead by a factor $\left(L_{d}-L_{s}\right)$.

Proof: Eq. (4) shows the relation between the length $L_{m}$ of the data, the length $\tilde{n}$ of the secret shares, and the design parameters $L_{d}$ and $L_{s}$. For $L_{d}-L_{s}>1$, the length of the secret shares $\tilde{n}$ is an integer fraction of the length $L_{m}$ of the data vector, and the communication overhead is reduced by a factor $\left(L_{d}-L_{s}\right)$.

Proposition 5 implies that the random shares $\boldsymbol{m}_{\boldsymbol{x}_{i}}^{(j)}$ that are induced by the code segments are less random compared to the baseline scheme.

\section{EXAMPLES}

In this section, we demonstrate the performance of the proposed scheme for a series of examples.

\section{A. Fully Connected Network with K Honest Clients}

Consider again the secure data aggregation protocol from Section II-C. It has been specified such that all secret shares are required to reconstruct the data and every client receives only one share from each other client. We set $L=K, L_{d}=K$, and $L_{s}=1$ and get the rate assignment $R_{0}=1$ and $R^{\prime}=1 / K$ and the secrecy rate $R=(K-1) / K$ for the wiretap code.

The clients encode their data as described above and generate $K$ secret shares $\boldsymbol{x}_{i}=\left[\boldsymbol{x}_{i}^{(0)}, \ldots, \boldsymbol{x}_{i}^{(K-1)}\right]$. The clients distribute the secret shares as specified in the protocol (i.e., the share $\boldsymbol{x}_{i}^{(j)}$ is shared by client $\mathrm{C}_{i}$ with client $\mathrm{C}_{j}$ by using a secure and authenticated link). After the distribution phase, every client $\mathrm{C}_{i}$ holds a set of shares $\left\{\boldsymbol{x}_{0}^{(i)}, \ldots, \boldsymbol{x}_{K-1}^{(i)}\right\}$, calculates the intermediate sum share

$$
\boldsymbol{x}_{\Sigma}^{(i)}=\sum_{j=0}^{K-1} \boldsymbol{x}_{j}^{(i)}
$$

and publishes (i.e., broadcasts) the result. The codeword $\boldsymbol{x}_{\Sigma}=$ $\left[\boldsymbol{x}_{\Sigma}^{(0)}, \ldots, \boldsymbol{x}_{\Sigma}^{(K-1)}\right]$ that encodes the sum of all data is obtained once all packets $\boldsymbol{x}_{\Sigma}^{(i)}$ are published, and the final result can be recovered as follows:

$$
\boldsymbol{H}_{1} \boldsymbol{x}_{\Sigma}^{T}=\boldsymbol{H}_{1}\left(\sum_{i=0}^{K-1} \boldsymbol{x}_{i}^{T}\right)=\sum_{i=0}^{K-1} \boldsymbol{m}_{i}^{T}=\boldsymbol{m}_{\Sigma}^{T} .
$$

In this protocol, reliability and perfect secrecy are guaranteed due to the choice of parameters of the employed wiretap codes. However, we require $K \geq 3$ since otherwise (i.e., for $K=2$ ) individual data of one client can be derived from the result and the data of the other client. We notice furthermore that compared to the baseline scheme the communication overhead is reduced by a factor $(K-1)$. In total,

$$
N_{A}=K^{2} /(K-1) L_{m}
$$

data symbols are exchanged. That is, for a large number of clients $K$ the communication overhead grows linearly with the 
number of clients as opposed to quadratic as for the baseline scheme $\left(N_{B L}=K^{2} L_{m}\right)$. We conclude that in the limit of large numbers of clients $K$ the proposed secret sharing scheme is asymptotically optimal in the sense that it minimizes the communication overhead since the number of symbols $N_{A}$ that are communicated equals the overall amount of data $N_{M}=$ $K L_{m}$ that is held by the clients.

\section{B. Fully Connected Network with M Cooperating Attackers}

In the previous example, we assumed that all clients in the network execute the protocol as specified and that clients do not cooperate in order to gain information on the data of other clients. Unfortunately, for the choice of code parameters in the previous example, information leakage occurs immediately if two or more clients cooperate and share their knowledge, and the leakage for $M$ cooperating adversaries is given by

$$
I_{M}=(M-1) /(K-1) L_{m} \log (q) .
$$

To maintain perfect secrecy in presence of an arbitrary set of $M$ cooperating clients, we update the design parameters as follows: $L=K, L_{d}=K$, and $L_{s}=M$, leading to the rate assignment $R_{0}=1$ and $R^{\prime}=M / K$ and a secrecy rate $R=(K-M) / K$ for the wiretap code. The protocol is then carried out exactly in the same way as in the previous example.

Reliability and secrecy are guaranteed by design as long as $M \leq K-2$ (i.e., as long as there is at least one other honest client). Compared to the previous example, the communication overhead is increased by a factor $(K-1) /(K-M)$, and

$$
N_{B}=K^{2} /(K-M) L_{m}
$$

data symbols are exchanged in total. However, compared to the baseline scheme the communication overhead is still reduced by a factor $(K-M)$. Due to the constraint $M \leq K-2$, we can conclude that our scheme reduces the communication overhead at least by a factor two. Furthermore, if we define $M$ to be a fraction $\alpha$ of the overall number of users (i.e., $M=\alpha K)$, the communication overhead scales linearly with the number of users and it is inversely proportional to $(1-\alpha)$.

\section{Erasure Recovery Capability}

In the last example, we highlight the erasure recovery capability of the proposed secret sharing scheme, and we investigate the impact of restricting the number of shares that are distributed among the clients. We assume that only $K^{\prime}<K$ shares per client are distributed in the network. Note that for $2 K^{\prime}<K-1$ the network is no longer required to be fully connected. We choose $L=K$ but leave $L_{s} \geq 1$ and $L_{d} \leq K$ unspecified for the moment. The protocol is now updated as follows: for every $j \in\{0, \ldots, K-1\}$, Client $\mathrm{C}_{j}$ distributes the shares $\left.\left\{\boldsymbol{x}_{j}^{\left(j-K^{\prime} \bmod K\right)}, \ldots, \boldsymbol{x}_{j}^{(j-1} \bmod K\right)\right\}$ and keeps the remaining shares secret. As before, the share $\boldsymbol{x}_{j}^{(i)}$ is sent by client $\mathrm{C}_{j}$ to client $\mathrm{C}_{i}$ using a secure link. After the distribution phase, every client $\mathrm{C}_{i}$ holds a set of shares $\left\{\boldsymbol{x}_{i}^{(i)}, \ldots, \boldsymbol{x}_{i+K^{\prime} \bmod K}^{(i)}\right\}$, calculates

$$
\boldsymbol{x}_{\Sigma, i}^{(i)}=\sum_{j=i}^{i+K^{\prime}} \boldsymbol{x}_{j}^{(i)},
$$

and forwards it securely to client $\mathrm{C}_{i-1}$. Client $\mathrm{C}_{i-1}$ then adds its share to the intermediate sum and forwards $\boldsymbol{x}_{\Sigma, i-1}^{(i)}=$ $\boldsymbol{x}_{\Sigma, i}^{(i)}+\boldsymbol{x}_{i-1}^{(i)}$ securely to client $\mathrm{C}_{i-2}$. The intermediate sum is updated and forwarded until after $K-K^{\prime}$ steps (including the initial step) the final result $\boldsymbol{x}_{\Sigma}^{(i)}=\boldsymbol{x}_{\Sigma, i-\left(K-K^{\prime}-1\right)}^{(i)} \bmod K$ is published by client $\mathrm{C}_{i-\left(K-K^{\prime}-1\right)} \bmod K$.

In this protocol only $L_{d}$ nodes need to initiate the calculation of an intermediate sum share since $L_{d}$ shares are sufficient to recover the final result. This case is illustrated in Table I for $K-K^{\prime}=3$ and $L_{d}=K-3$. Here, shares that are kept secret are shaded in grey. On the other hand, if all nodes participate, the final result can be recovered from any subset of $L_{d}$ intermediate sum shares. This may be beneficial, if the calculation in the network is carried out over a longer period in time, and not all clients are available during the data collection (e.g., due to sleep cycles).

In this protocol, secrecy is maintained for $L_{s} \geq 1$. In the data distribution phase, every client receives only one share from other clients. In the calculation phase, client $\mathrm{C}_{j}$ cannot draw any conclusions on the share that client $\mathrm{C}_{j+1} \bmod K$ contributed to the intermediate sum $\boldsymbol{x}_{\Sigma, j+1}^{(i)} \bmod K$ as long as the share $\boldsymbol{x}_{\Sigma, j+2}^{(i)} \bmod K$ does not leak out. In the last step, the second to last client $\mathrm{C}_{i-\left(K-K^{\prime}-2\right)} \bmod K$ can recover the share $\boldsymbol{x}_{i-\left(K-K^{\prime}-1\right) \bmod K}$ of client $\mathrm{C}_{i-\left(K-K^{\prime}-1\right) \bmod K}$ by combining the final published result with its forwarded intermediate sum share. However, due to the way the connections were set in this example, it is the first share that it observes from that client. Accordingly, (i) every client observes at most one share from any other client (i.e., we can set $L_{s}=1$ ), and (ii) effectively $K^{\prime}+1$ shares are communicated to other clients in the network. Unfortunately, the analysis for $M$ cooperating users becomes quite involved in this case. It is hence omitted.

To evaluate the communication overhead, we consider two different cases. In the first case, if all $K$ clients participate in the calculation phase, the overall number of symbols that need to be communicated is given by (for $L_{s}=1$ )

$$
N_{C}^{(1)}=K^{2} /\left(L_{d}-1\right) L_{m}
$$

We can see that it is independent of the parameter $K^{\prime}$, and for $L_{d}=K$, it leads to the same communication overhead as in the first example. If we wish to reconstruct the result based on a fraction $\rho$ of the shares, we can set $L_{d}=\rho K$. As long as $\rho K \gg 1$, the communication overhead scales linearly with the number of clients $K$ and it is inversely proportional to $\rho$.

In the second case, we assume that only $L_{d}<K$ clients participate in the calculation phase. The number of symbols that are communicated in the protocol is given by

$$
N_{C}^{(2)}=\left(K^{2}-\left(K-L_{d}\right)\left(K-K^{\prime}\right)\right)\left(L_{m} /\left(L_{d}-1\right)\right)
$$

where the first term of the product corresponds to the number of shares that are exchanged in total and the second term is the number of symbols per share. Clearly, the number of transmissions is reduced compared to the previous schemes while the length of the shares has been increased. To minimize the communication overhead, we set $K^{\prime}=1$. In this case, the fully connected network turns into a ring. If we now again wish to reconstruct the result based on a fraction $\rho$ of the 
TABLE I. ENCODING AND DISTRIBUTION OF DATA PACKETS AND COMPUTATION OF INTERMEDIATE SUM SHARES FOR $K-K^{\prime}=3$ AND $L_{d}=K-3$.

\begin{tabular}{|c|c|c|c|c|c|c|c|c|}
\hline & \multicolumn{8}{|c|}{ Knowledge obtained by $\mathrm{C}_{i}$} \\
\hline \multirow{9}{*}{$\begin{array}{l}\tilde{d} \\
\delta \\
\delta \\
\tilde{0} \\
ن\end{array}$} & \multirow{9}{*}{$\begin{array}{l}\boldsymbol{x}_{0}^{(0)} \\
\boldsymbol{x}_{1}^{(0)} \\
\boldsymbol{x}_{2}^{(0)} \\
\vdots \\
\vdots \\
\boldsymbol{x}_{K-3}^{(0)} \\
\boldsymbol{x}_{K-2}^{(0)} \\
\boldsymbol{x}_{K-1}^{(0)}\end{array}$} & \multirow{3}{*}{$\begin{array}{l}\boldsymbol{x}_{0}^{(1)} \\
\boldsymbol{x}_{1}^{(1)} \\
\boldsymbol{x}_{(1)}^{(1)}\end{array}$} & \multirow{3}{*}{$\begin{array}{l}\boldsymbol{x}_{0}^{(2)} \\
\boldsymbol{x}_{1}^{(2)} \\
\boldsymbol{x}_{2}^{(2)}\end{array}$} & \multirow{3}{*}{$\begin{array}{l}\boldsymbol{x}_{0}^{(3)} \\
\boldsymbol{x}_{1}^{(3)} \\
\boldsymbol{x}_{2}^{(3)}\end{array}$} & \multirow{3}{*}{$\begin{array}{l}\cdots \\
\boldsymbol{x}_{1}^{(4)} \\
\boldsymbol{x}_{2}^{(4)}\end{array}$} & \multirow{3}{*}{$\begin{array}{l}\cdots \\
\cdots \\
\boldsymbol{x}_{2}^{(5)}\end{array}$} & \multirow{2}{*}{$\cdots$} & \multirow{3}{*}{$\begin{array}{l}\boldsymbol{x}_{0}^{(K-1)} \\
\boldsymbol{x}_{1}^{(K-1)} \\
\boldsymbol{x}_{2}^{(K-1)}\end{array}$} \\
\hline & & & & & & & & \\
\hline & & & & & & & $\ldots$ & \\
\hline & & & $\ddots$ & $\ddots$ & $\ddots$ & $\ddots$ & & \\
\hline & & & & & & & & \\
\hline & & & : & : & $\ddots$ & $\ddots$ & $\ddots$ & $\ddots$ \\
\hline & & & & $\ldots$ & & $\boldsymbol{x}_{K-3}^{(K-3)}$ & $\boldsymbol{x}_{K-3}^{(K-2)}$ & $\boldsymbol{x}_{K-3}^{(K-1)}$ \\
\hline & & $\boldsymbol{x}_{K-2}^{(1)}$ & & & & & $\boldsymbol{x}_{K-2}^{(K-2)}$ & $\boldsymbol{x}_{K-2}^{(K-1)}$ \\
\hline & & $\boldsymbol{x}_{K-1}^{(1)}$ & $\boldsymbol{x}_{K-1}^{(2)}$ & $\ldots$ & $\ldots$ & $\ldots$ & $\cdots$ & $\boldsymbol{x}_{K-1}^{(K-1)}$ \\
\hline $\begin{array}{l}\mathrm{C}_{i} \text { com- } \\
\text { putes } \\
\text { and for- } \\
\text { wards }\end{array}$ & & & & $\boldsymbol{x}_{\Sigma, 3}^{(3)}$ & & $\boldsymbol{x}_{\Sigma, K-3}^{(K-3)}$ & $\boldsymbol{x}_{\Sigma, K-2}^{(K-2)}$ & $\boldsymbol{x}_{\Sigma, K-1}^{(K-1)}$ \\
\hline & & & $\boldsymbol{x}_{\Sigma, 2}^{(3)}$ & & $\boldsymbol{x}_{\Sigma, K-4}^{(K-3)}$ & $\boldsymbol{x}_{\Sigma, K-3}^{(K-2)}$ & $\boldsymbol{x}_{\Sigma, K-2}^{(K-1)}$ & \\
\hline $\begin{array}{c}\mathrm{C}_{i} \text { pub- } \\
\text { lishes }\end{array}$ & & $\boldsymbol{x}_{\Sigma}^{(3)}$ & $\ldots$ & $\boldsymbol{x}_{\Sigma}^{(K-3)}$ & $\boldsymbol{x}_{\Sigma}^{(K-2)}$ & $\boldsymbol{x}_{\Sigma}^{(K-1)}$ & & \\
\hline $\begin{array}{c}\mathrm{C}_{i} \\
\text { learns }\end{array}$ & & & $\boldsymbol{x}_{1}^{(3)}$ & $\ldots$ & $\boldsymbol{x}_{K-5}^{(K-3)}$ & $\boldsymbol{x}_{K-4}^{(K-2)}$ & $\boldsymbol{x}_{K-3}^{(K-1)}$ & \\
\hline
\end{tabular}

shares, we can set $L_{d}=\rho K$. For $K^{\prime}=1$ and $\rho K \gg 1$, we get $N_{C}^{(2)} \approx K L_{m}=N_{M}$. That is, for sufficiently large $K$ the redundancy introduced by the secret sharing can be utilized to reduce the number of transmissions while keeping the overall communication overhead constant.

\section{CONCLUSIONS}

In this paper, we have presented a novel secret sharing scheme that guarantees perfect secrecy and that is constructed based on coding techniques for the wiretap channel. Due to the linearity of the considered code families, the secret sharing scheme is applicable to secure multi-party computation if the goal is to calculate linear functions of data that are distributed in a network. For the examples that we discussed in this paper, we showed that the communication overhead for our scheme scales linearly with number of clients. This leads to a significant reduction in communication costs compared to conventional schemes for which the communication overhead grows quadratically with the number of clients. This performance has been achieved by employing nested ReedSolomon codes to implement the underlying wiretap codes. While in this paper we have only considered the case of passive eavesdropping, the proposed framework can also be applied in cases where clients tamper with the data given that the effect of such attacks can be modelled in the design of the underlying wiretap codes. This can be achieved by adopting, e.g., polar code designs (see, e.g., [6]-[8]) to the resulting wiretap channel model. However, in contrast to the scheme proposed in this paper, this approach would not provide perfect secrecy for finite block length.

\section{REFERENCES}

[1] Z. Erkin, J. Troncoso-Pastoriza, R. Lagendijk, and F. Perez-Gonzalez, "Privacy-preserving data aggregation in smart metering systems: An overview," IEEE Signal Processing Magazine, vol. 30, no. 2, pp. 7586,2013
[2] P. Paillier, "Public-key cryptosystems based on composite degree residuosity classes," in Advances in cryptology - EUROCRYPT'99. Springer, 1999, pp. 223-238.

[3] R. Lagendijk, Z. Erkin, and M. Barni, "Encrypted signal processing for privacy protection: Conveying the utility of homomorphic encryption and multiparty computation," IEEE Signal Processing Magazine, vol. 30, no. 1, pp. 82-105, 2013.

[4] A. Wyner, "The wire-tap channel," The Bell System Technical Journal, vol. 54 , no. 8, pp. 1355-1387, 1975.

[5] A. Subramanian and S. McLaughlin, "MDS codes on the erasureerasure wiretap channel," arXiv preprint arXiv:0902.3286, 2009.

[6] M. Andersson, V. Rathi, R. Thobaben, J. Kliewer, and M. Skoglund, "Nested polar codes for wiretap and relay channels," IEEE Communications Letters, vol. 14, no. 8, pp. 752-754, 2010.

[7] H. Mahdavifar and A. Vardy, "Achieving the secrecy capacity of wiretap channels using polar codes," IEEE Transactions on Information Theory, vol. 57, no. 10, pp. 6428-6443, 2011.

[8] E. Sasoglu and A. Vardy, "A new polar coding scheme for strong security on wiretap channels," in 2013 IEEE International Symposium on Information Theory Proceedings (ISIT), 2013, pp. 1117-1121.

[9] R. McEliece and D. Sarwate, "On sharing secrets and Reed-Solomon codes," Communications of the ACM, vol. 24, no. 9, pp. 583-584, 1981.

[10] A. Shamir, "How to share a secret," Communications of the ACM, vol. 22, no. 11, pp. 612-613, 1979.

[11] S. El Rouayheb, E. Soljanin, and A. Sprintson, "Secure network coding for wiretap networks of type II," IEEE Transactions on Information Theory, vol. 58, no. 3, pp. 1361-1371, 2012.

[12] S. Obana, "Almost optimum t-cheater identifiable secret sharing schemes," in Advances in Cryptology-EUROCRYPT 2011. Springer, 2011, pp. 284-302.

[13] A. Cevallos, S. Fehr, R. Ostrovsky, and Y. Rabani, "Unconditionallysecure robust secret sharing with compact shares," in Advances in Cryptology-EUROCRYPT 2012. Springer, 2012, pp. 195-208.

[14] R. Xu, K. Morozov, and T. Takagi, "On cheater identifiable secret sharing schemes secure against rushing adversary." in Advances in Information and Computer Security-IWSEC. Springer, 2013, pp. 258271. 